\title{
Endoscopic Resection of Vestibular Schwannomas
}

\author{
Pradeep Setty ${ }^{1}$ Kenneth P. D’Andrea ${ }^{1} \quad$ Emily Z. Stucken ${ }^{2}$ Seilesh Babu ${ }^{2}$ Michael J. LaRouere ${ }^{2}$ \\ Daniel R. Pieper ${ }^{3}$
}
1 Section of Neurosurgery, St John Providence Health System, Michigan State Unviersty, Southfield, Michigan, United States
2 Department of Neurotology, Michigan Ear Institute, Farmington Hills, Michigan, United States
${ }^{3}$ Department of Neurosurgery, Oakland University William Beaumont School of Medicine, Royal Oak, Michigan, United States

Address for correspondence Pradeep Setty, DO, 180 S. Georgetown Square, Royal Oak, MI 48067, United States (e-mail: DrPSetty@gmail.com).

J Neurol Surg B 2015;76:230-238.

\begin{abstract}
Objective To report our results and the technical details of fully endoscopic resection of vestibular schwannomas.

Design Prospective observational study.

Setting A single academic institution involving neurosurgery and neurotology.

Participants Twelve consecutive patients who underwent fully endoscopic resection of a vestibular schwannoma.

Main Outcome Measures Hearing preservation, based on the American Association of Otolaryngology-Head and Neck Surgeons (AAO-HNS) score as well as the Gardener and Robertson Modified Hearing Classification (GR). Facial nerve preservation based on the House-Brackmann (HB) score.

Keywords

- acoustic neuroma

- cerebellopontine angle

- endoscopy

- skull base

- vestibular schwannoma

Results All patients successfully underwent gross total resection. Facial nerve preservation rate was $92 \%$ with 11 of 12 patients retaining an $\mathrm{HB}$ score of $1 / 6$ postoperatively. Hearing preservation rate was $67 \%$ with 8 of 12 patients maintaining a stable AAO-HNS grade and GR score at follow-up. Mean tumor size was $1.5 \mathrm{~cm}$ (range: 1-2 cm). No patients experienced postoperative cerebrospinal fluid leak, infection, or cranial nerve palsy for a complication rate of $0 \%$. Mean operative time was 261.6 minutes with an estimated blood loss of $56.3 \mathrm{~mL}$ and average length of hospital stay of 3.6 days.

Conclusion A purely endoscopic approach is a safe and effective option for hearing preservation surgery for vestibular schwannomas in appropriately selected patients.
\end{abstract}

\section{Introduction}

In 1879 Max Nitze first described the use of the operative endoscope, ${ }^{1}$ but it was not until 1917 that it was described for cerebellopontine angle (CPA) surgery. In that instance, Doyen utilized the surgical endoscope to perform a trigeminal neurectomy, and thus began the road to endoscopy in the posterolateral skull base. ${ }^{2}$ These initial endoscopes were crudely designed,

received

September 30, 2014

accepted after revision

November 16, 2014

published online

January 21, 2015 however, and provided little in the way of light or magnification and therefore never gained much popularity. The advent of the operative microscope in the 1960s proved the endoscope to be obsolete at the time. In the 1990s, however, improved technology led to the reintroduction of endoscopy to skull base surgery. Over the past 2 decades, the popularity of endoscopic skull base surgery has exploded; however, most of it has been limited to endonasal approaches of the anterior and middle fossa. (c) 2015 Georg Thieme Verlag KG Stuttgart · New York
DOI http://dx.doi.org/ 10.1055/s-0034-1543974. ISSN 2193-6331. 
Subsequently, the operative microscope has continued to be the workhorse of posterolateral skull base surgery. But the microscope utilizes a linear light source that is outside the surgical field that struggles to provide optimal visualization around the intricate corners of the CPA. As a result, surgeons are forced to use larger cranial openings, cerebellar retraction, and manipulation of neurovascular structures, all of which can increase morbidity and lengthen patient recovery, to compensate for the lack of illumination.

In recent years, some skull base surgeons have begun experimenting with endoscopy in the CPA. Several reports have described the superior visualization of the neurovascular structures of the CPA with endoscopy compared with microscopy. ${ }^{3-9}$ However, logistical barriers have prevented the endoscope from replacing the microscope but rather have relegated it to an adjunct role. As a result, endoscopically assisted procedures started to gain traction in the posterolateral skull base. More recently, as several of these logistical barriers have been overcome with the introduction of pneumatic holding arms, some authors have had success with fully endoscopic microvascular decompressions in the CPA. ${ }^{10-15}$ However, reports of purely endoscopic tumor resection, especially vestibular schwannoma, in the CPA have been scarce. We report results of our purely endoscopic technique to resect vestibular schwannomas.

\section{Methods}

Between 2006 and 2013, 12 patients underwent a fully endoscopic resection of a vestibular schwannoma at our institution. A prospective observational study was performed to gather pre-, post-, and perioperative data that were then analyzed and are presented in this study. Patients initially presented with a variety of different symptoms, and all underwent gadolinium-enhanced magnetic resonance imaging (MRI) of the brain where a unilateral vestibular schwannoma, later confirmed by pathology, was discovered. No patients had bilateral tumors; nor did any have additional intracranial findings. All 12 patients underwent audiometric testing, in which salvageable hearing was confirmed present. A full neurologic examination was also performed including detailed cranial nerve findings. In addition, tumor size was determined by measuring the maximum diameter in both the axial and coronal slices.

All patients underwent hearing preservation surgery for resection of vestibular schwannoma in a fully endoscopic technique via retrosigmoid craniectomy. Perioperative data such as estimated blood loss, operative time, and length of hospital stay were recorded. Extent of tumor resection was determined through both intraoperative visualization as well as postoperative gadolinium-enhanced MRI. Follow-up ranged from 6 to 12 months. Facial nerve preservation was evaluated both pre- and postoperatively, and reported utilizing the House-Brackmann (HB) grading system. All patients underwent audiometric testing before surgery as well as at follow-up including air conduction pure tone average (AC PTA), expressed in decibels, and Word Recognition Score (WRS), expressed as a percentage. The AC PTA and WRS were analyzed to determine a pre- and postoperative hearing score, based on two scoring systems: the Committee on
Table 1 Committee on hearing and equilibrium guidelines for the evaluation of hearing preservation in Acoustic Neuroma Classification System (AAO-HNS grade) ${ }^{16}$

\begin{tabular}{|l|l|l|}
\hline Class & PTA, dB & WRS, \% \\
\hline A & $\leq 30 \mathrm{~dB}$ and & $\geq 70$ \\
\hline B & $>30 \mathrm{~dB}, \leq 50 \mathrm{~dB}$, and & $\geq 50$ \\
\hline C & $>50 \mathrm{~dB}$ and & $\geq 50$ \\
\hline D & Any level & $<50$ \\
\hline
\end{tabular}

Abbreviations: AAO-HNS, Association of Otolaryngology-Head and Neck Surgeons; PTA, pure tone average; WRS, Word Recognition Score.

Hearing and Equilibrium Guidelines for the Evaluation of Hearing Preservation in Acoustic Neuroma (AAO-HNS), ${ }^{16}$ which is detailed in - Table 1, and the Gardener and Robertson Modified Hearing Classification, listed in -Table 2. Cranial neuropathies including facial numbness, diplopia, and dysphagia were recorded. In addition, all complications such as cerebrospinal fluid (CSF) leak, wound infection, and worsened symptomatology were listed.

\section{Technical Note}

\section{Operative Setup}

All patients undergo general anesthesia and endotracheal intubation. Positioning is supine with the head elevated to 30 degrees and rotated to the contralateral side. It is important to create enough rotation to allow the surgeon access to the retrosigmoid suboccipital corridor while not going so far as to compress the jugular veins and restrict venous outflow. The positioning allows gravity to aid in exposing the ipsilateral cerebellar hemisphere that will naturally fall away from the petrous portion of the temporal bone, maximizing the corridor to the CPA. No paralytics can be used because facial nerve monitoring is necessary, and therefore immobilization of the head with pins and a head holder is mandatory. Without this step, movement of the patient during the procedure can cause catastrophic trauma because it is possible that the endoscope, which is fixed to the operative table instead of being held by hand, can impale the brainstem.

Once the patient is adequately positioned, the polyaxial pneumatic holding arm (-Fig. 1) is attached to the

Table 2 Gardener and Robertson Modified Hearing Classification

\begin{tabular}{|l|l|l|l|}
\hline Class & Description & PTA, dB & WRS, \% \\
\hline I & Good to excellent & $0-30$ & $70-100$ \\
\hline II & Serviceable & $31-50$ & $50-69$ \\
\hline III & Nonserviceable & $51-90$ & $5-49$ \\
\hline IV & Poor & $91-$ maximum & $1-4$ \\
\hline V & None & Not testable & 0 \\
\hline
\end{tabular}

Abbreviations: PTA, pure tone average; WRS, Word Recognition Score. 


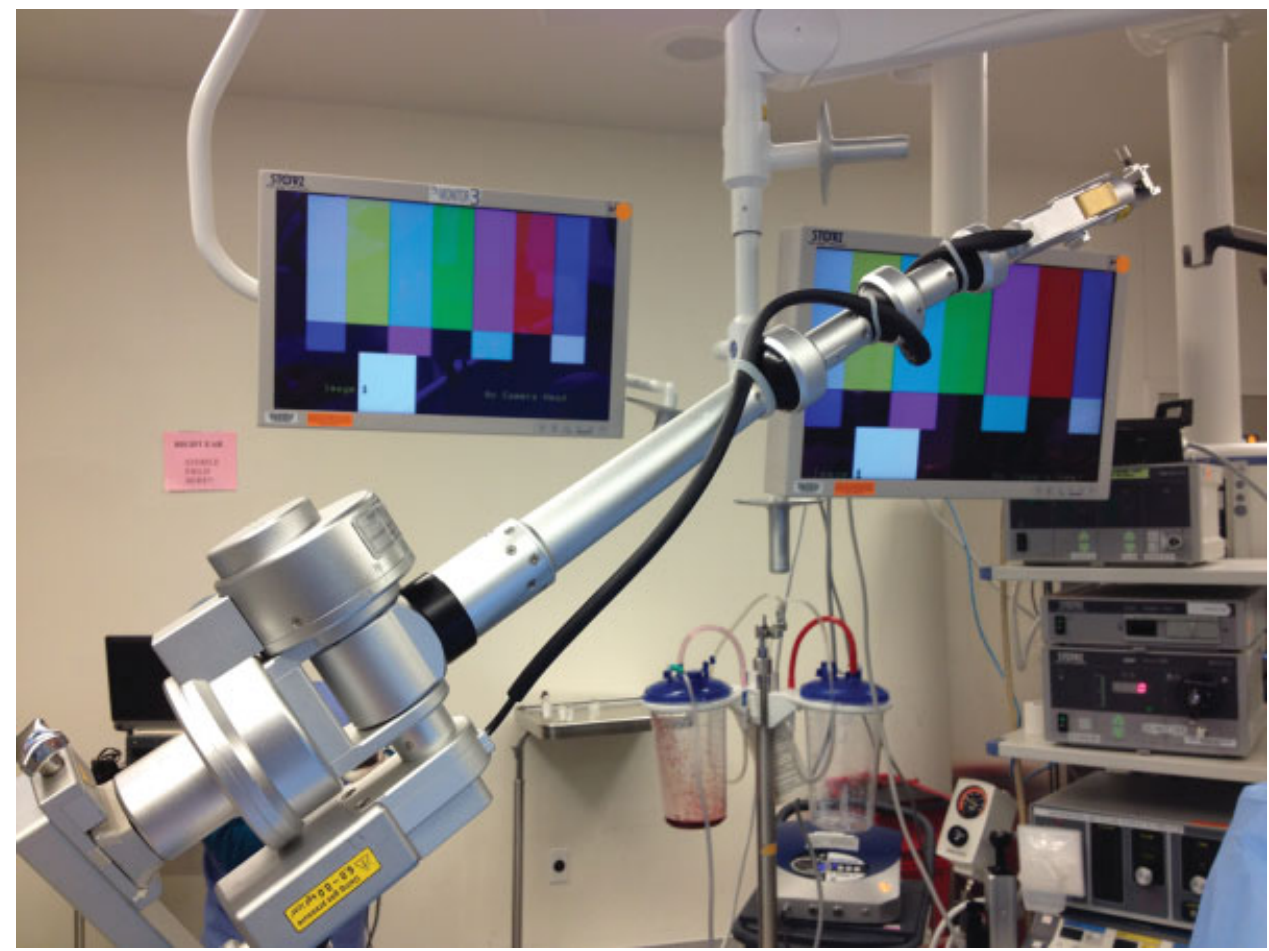

Fig. 1 Polyaxial pneumatic holding arm.

contralateral side of the operating table, allowing it to arch over the patient's head. This location is strategically chosen to allow the holding arm to secure the endoscope in place while minimizing obstruction of the operative field. The highdefinition monitor is placed on the contralateral side of the patient, in a direct line-of-sight from where the primary surgeon stands, allowing for appropriate visualization without significant movement (-Fig. 2). Next the ipsilateral transverse sinus is approximated by drawing a line between the root of the zygoma and the external occipital

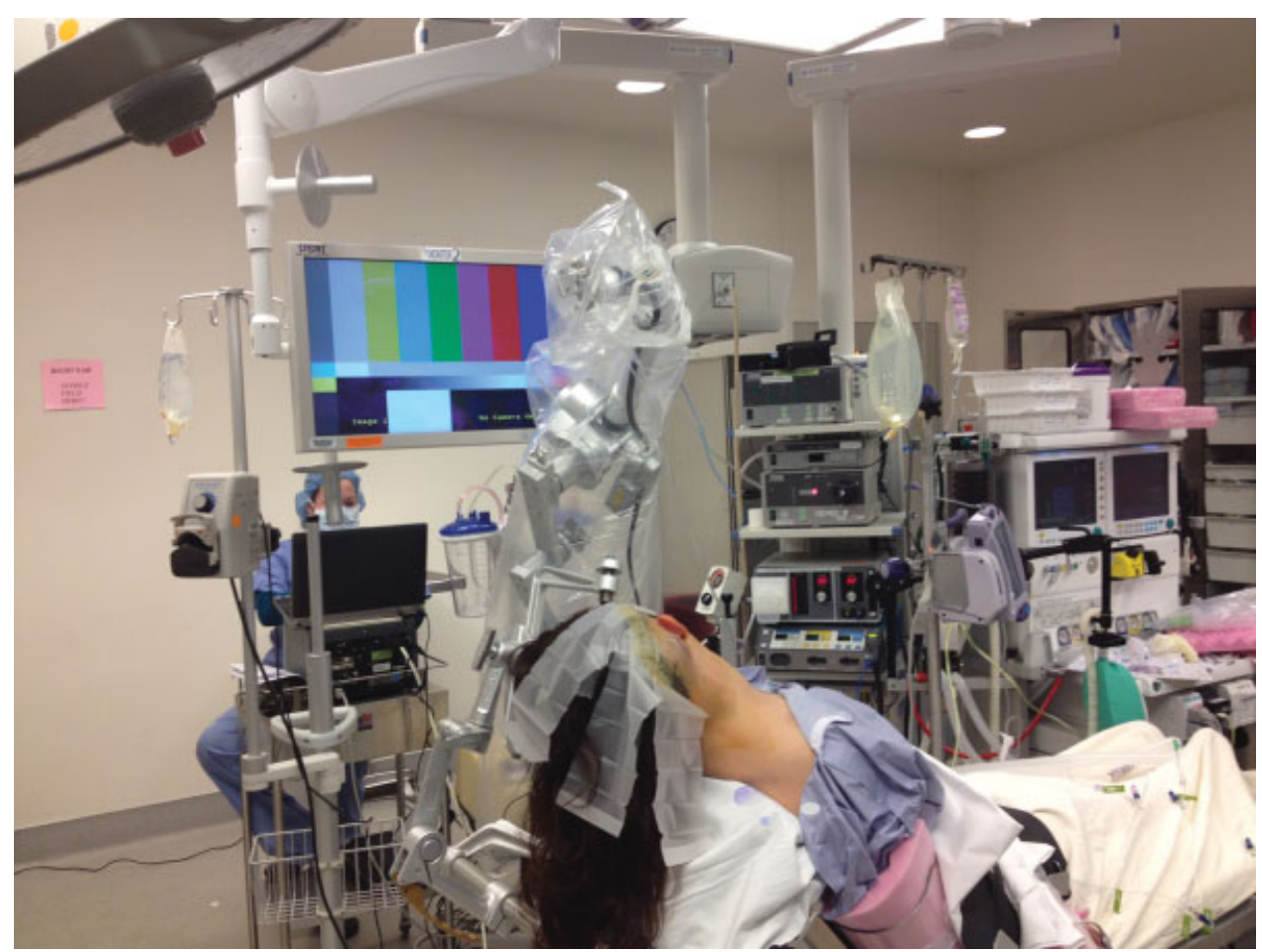

Fig. 2 Preoperative setup with the patient in the appropriate position. The holding arm is mounted to the bed on the contralateral side, and the high-definition monitor is directly behind it. 


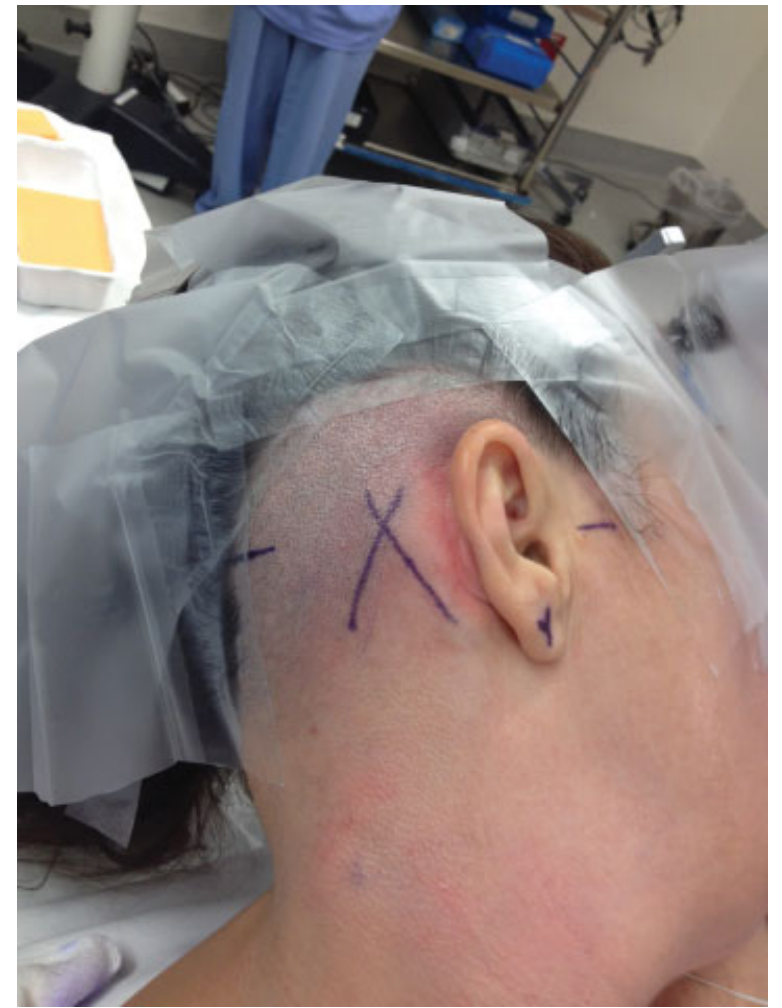

Fig. 3 Preoperative view of skin markings prior to incision. Posteriorly, the external occipital protuberance has been marked with a horizontal line. The estimated location of the sigmoid sinus has also been marked with a crossing line that delineates the patient's hairline. Anteriorly, the root of the zygoma has been marked with a horizontal line.

protuberance. The sigmoid sinus is then approximated parallel and lateral to the posterior mastoid groove (-Fig. 3). The incision is then marked over the intersection of these two lines, parallel to the hairline. Facial nerve monitoring and brainstem auditory evoked response are routinely used for monitoring, eliminating the possibility of paralytic use by anesthesia.

\section{Operative Details}

Before incision, brain relaxation is encouraged by giving 1 to 2 $\mathrm{g} / \mathrm{kg}$ of mannitol, $20 \mathrm{mg}$ furosemide, and decreasing partial pressure of carbon dioxide to $28 \mathrm{~mm} \mathrm{Hg}$. A small linear incision is made at the previously marked site over the approximated transverse-sigmoid (TS) junction. Monopolar cautery is used to dissect down to the calvarium, and retractors assist in providing exposure. The asterion and occipitomastoid suture are key landmarks and should be identified. At this point a high-speed drill is used to create a $14-\mathrm{mm}$ craniectomy inferior to the asterion and one-third anterior, two-thirds posterior to the occipitomastoid suture (-Fig. 4). This location allows for skeletonization of the TS junction in the superolateral corner of the defect. Bone wax is used to control bleeding emissary veins and obtain adequate hemostasis. A curvilinear dural opening is made along the edge of the TS junction.

Once the dural flap is reflected medially and inferiorly, a 4-mm 0-degree endoscope, secured to the holding arm, is introduced and advanced in the corridor between the

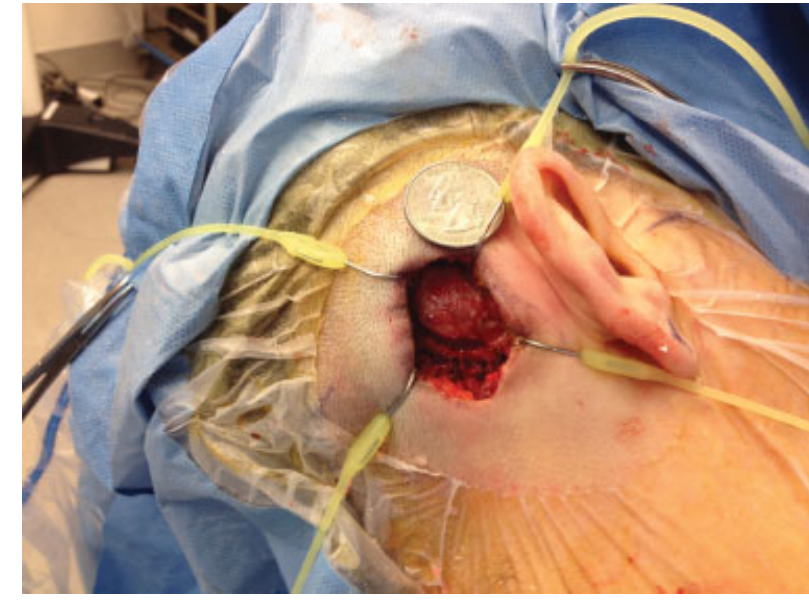

Fig. 4 Intraoperative view of the size of the retrosigmoid craniotomy. A U.S. quarter is placed just above the incision for reference size.

cerebellar hemisphere and petrous temporal bone. CSF is continually suctioned, allowing for more brain relaxation and increased visualization. The petrosal vein is encountered, coagulated, and cut, allowing for further mobilization of the cerebellum. Once the prepontine cistern is reached, CSF egress allows for full brain relaxation and opens the CPA for optimal visualization. The endoscope is then retracted and the tumor is identified and inspected until the cochlear nerve is identified. Angled endoscopes, such as the 30-degree scope, can be utilized to inspect ventrally to the tumor, identifying the location of the facial nerve. Frequent irrigation through an attached sheath clears debris and acts as a coolant, preventing thermal damage to adjacent structures.

At this point, the tumor capsule is opened and internal debulking of the intracapsular contents is performed. The remaining tumor is then mobilized away from the facial and cochlear nerves, and removal is continued until gross total resection is achieved. Facial nerve-stimulating probes are frequently used throughout the procedure to avoid damage and ensure preservation. Once resection is completed, the endoscope again inspects the tumor bed from the brainstem to the porus acusticus to ensure that no residual tumor remains. The 30-degree endoscope can be used to confirm that all tumor has been removed from the internal auditory canal (IAC).

If tumor does remain, the IAC can be drilled out to allow for complete tumor resection. Meticulous hemostasis is necessary. However, if significant bleeding does occur, it should be controlled with gentle pressure or bipolar cautery. It is essential to avoid removing the endoscope in the event of bleeding because recannulating the CPA corridor will be difficult. Once the procedure is completed, watertight dural closure is completed (-Fig. 5). The bony defect is then filled with bone cement (-Fig. 6), and the skin is closed in layers with absorbable sutures.

\section{Results}

- Table 3 lists the baseline characteristics on all 12 patients who underwent fully endoscopic resection of vestibular 


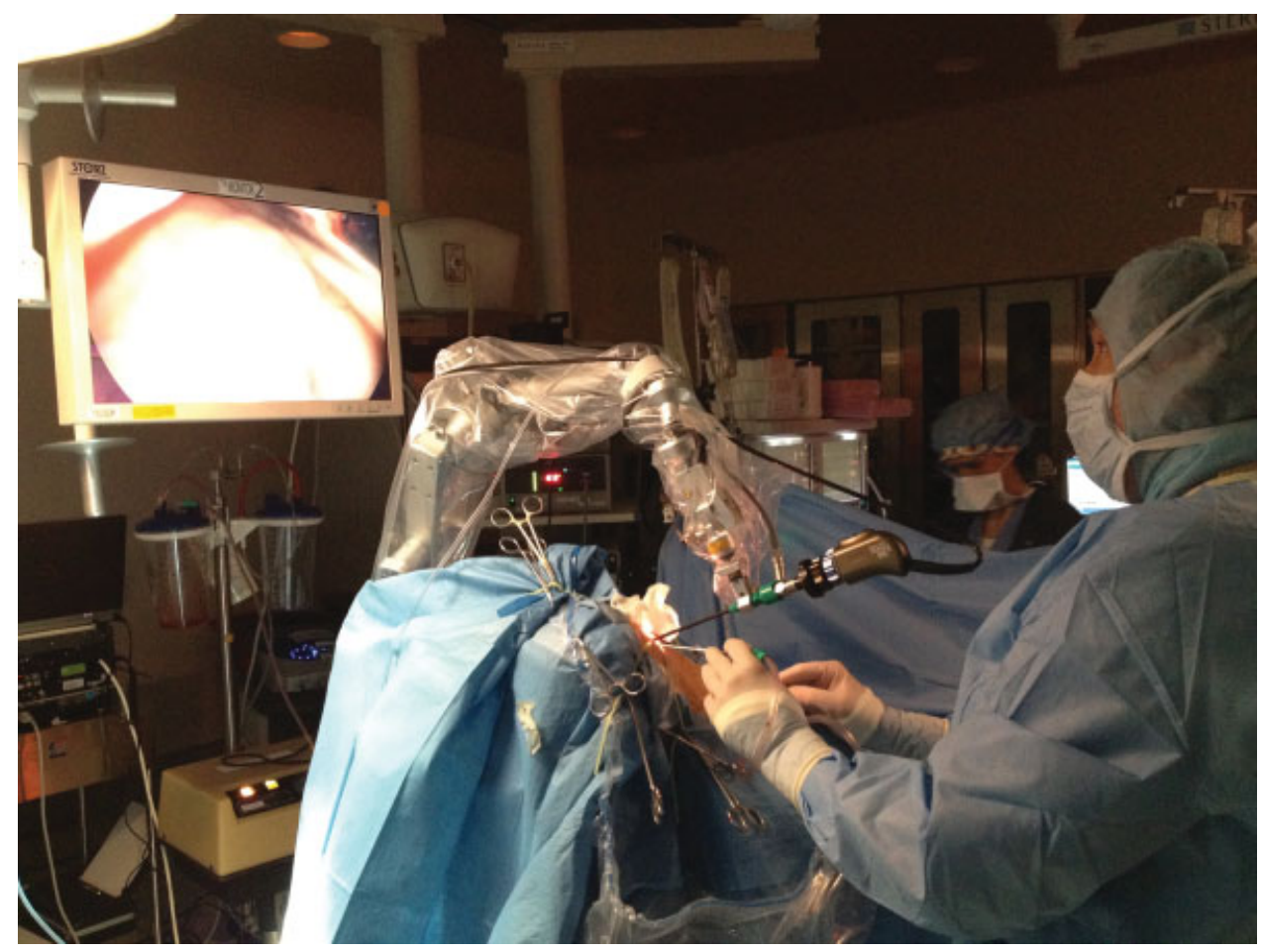

Fig. 5 View of dural closure with dural onlay graft.

schwannoma. Eight patients were male; four were female. All tumors were unilateral with five on the right side and seven on the left side. The mean age at the time of surgery was 46.7 years (range: 28-68 years). The most common presenting

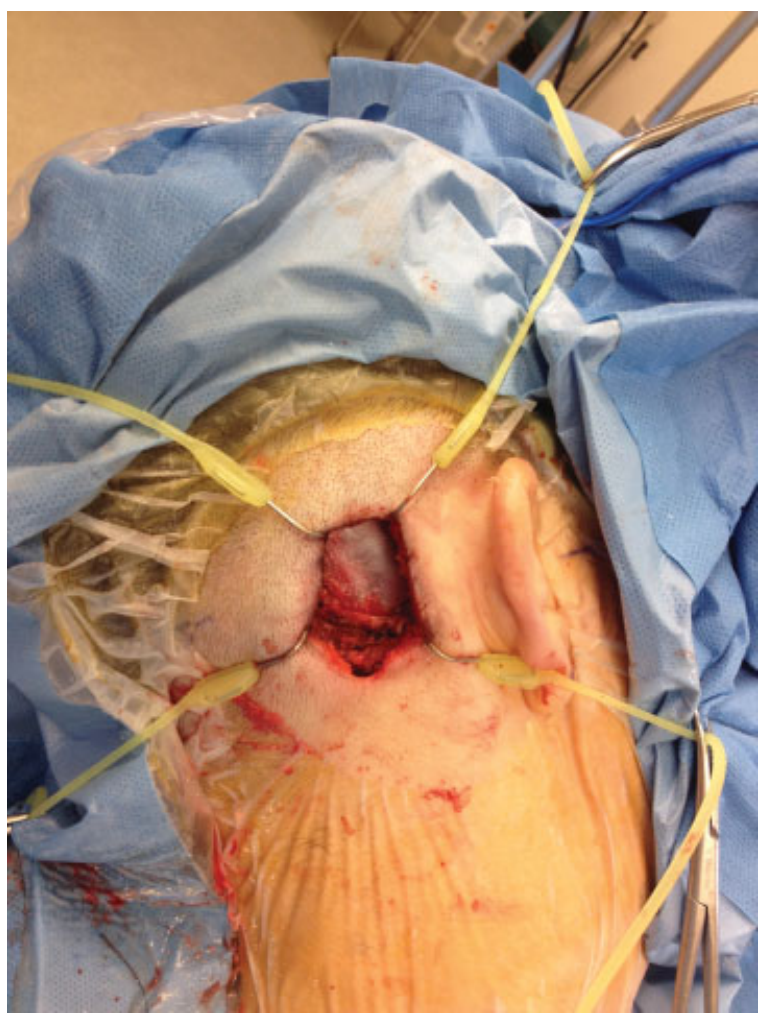

Fig. 6 View of craniectomy defect filled in with hydroxyapatite bone cement. symptom was tinnitus, which was present in 6 of 12 patients (50\%). Three patients (25\%) presented with vertigo, two patients $(17 \%)$ presented with hearing decline, and one patient $(8 \%)$ presented with facial pain. No patients were found to have any other cranial nerve deficits or complaints before surgery.

\section{Tumor Resection}

The mean tumor size at the time of surgery was $1.5 \mathrm{~cm}$ (range: $1.0-2.0 \mathrm{~cm}$ ). All 12 patients (100\%) underwent successful

Table 3 Patient characteristics

\begin{tabular}{|l|l|}
\hline Total patients & 12 \\
\hline Age, $\mathbf{y}$ & \\
\hline Mean & 46.7 \\
\hline Range & $28-68$ \\
\hline Gender (\%) & \\
\hline Male & $8(67)$ \\
\hline Female & $4(33)$ \\
\hline Side of tumor (\%) & \\
\hline Right & $5(42)$ \\
\hline Left & $7(58)$ \\
\hline Presenting symptom (\%) & \\
\hline Tinnitus & $6(50)$ \\
\hline Vertigo & $3(25)$ \\
\hline Hearing difficulty & $2(17)$ \\
\hline Facial pain & $1(8)$ \\
\hline
\end{tabular}


gross total resection as seen both intraoperatively as well as on postoperative imaging. One patient ( $8 \%)$, however, did have tumor recurrence in the distal IAC 2 years later. This patient subsequently refused both radiosurgery as well as reresection via a middle fossa approach and chose to undergo a translabyrinthine approach. The remaining 11 patients (92\%) had no recurrence at follow-up. Data are listed in - Table 4.

\section{Facial Nerve Preservation}

All 12 patients had full function of the facial nerve preoperatively (HB: 1/6). Intraoperatively, anatomical preservation of the facial nerve was successful in all patients (100\%). Postoperatively, 11 of 12 patients (92\%) retained full facial nerve function (HB: $1 / 6)$. One patient (8\%) did experience some postoperative facial weakness (HB: 3/6) that continued through follow-up. Data are presented in - Table 4.

\section{Hearing Preservation}

Hearing preservation grading was based on the AAOHNS score, detailed in - Table 1, and the Gardener and Robertson Modified Hearing Classification, listed in - Table 2. Patients underwent full pre- and postoperative audiometric testing including AC PTA and WRS. This data was used to create a preand postoperative grade. These grades were then compared to determine if a patient's hearing remained stable or worsened after surgery. Overall, 8 of 12 patients (67\%) had stable hearing grade postoperatively; 4 patients (33\%) worsened based on both scoring systems. According to the AAO-HNS grading system, preoperatively five patients were graded A, six were graded $B$, one was graded $C$, and no patients were graded D. Postoperatively, three patients were graded A, five were graded B, zero were graded C, and four were graded D. The Gardener and Robertson Modified Hearing Classification demonstrated preoperatively that five patients were class I, six patients were class II, and one patient was class III. Postoperatively, three patients were grade I, five patients were grade II, and four patients were grade V. Full hearing data are listed in - Table 4 .

\section{Perioperative Data}

The mean length of operative time was 261.6 minutes (range: 180-512 minutes). Length of stay ranged from 2 to 7 days with a mean of 3.6 days. All patients spent the first night postoperatively in the intensive care unit and subsequent days in regular patient beds. Most of the patients were discharged by postoperative day 4 , with only two patients remaining past this mark. Estimated blood loss ranged between 50 and $100 \mathrm{~mL}$ with a mean of $56.3 \mathrm{~mL}$. No patients needed any time of significant rehabilitation stay; all were discharged home from the hospital. Data are listed in - Table 5.

\section{Complications}

No significant complications occurred intraoperatively. This includes zero CSF leaks, wound infections, abscesses, or medical complications. Additionally, no new neurologic deficits or cranial neuropathies were discovered postoperatively

\begin{tabular}{|c|c|c|c|c|c|c|c|c|c|c|c|c|}
\hline 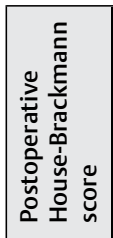 & $\stackrel{\odot}{2}$ & $\stackrel{\circ}{?}$ & $\stackrel{0}{?}$ & $\stackrel{6}{=}$ & $\frac{\varphi}{m}$ & $\stackrel{\circ}{=}$ & $\stackrel{0}{=}$ & $\stackrel{0}{\rightleftharpoons}$ & $\stackrel{\bullet}{\rightleftharpoons}$ & $\stackrel{\varphi}{=}$ & $\stackrel{\varphi}{=}$ & $\stackrel{6}{?}$ \\
\hline 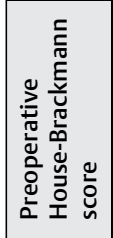 & $\stackrel{0}{=}$ & $\stackrel{\varphi}{?}$ & $\stackrel{6}{7}$ & $\stackrel{0}{=}$ & $\stackrel{\varphi}{r}$ & $\stackrel{0}{=}$ & $\stackrel{\bullet}{=}$ & $\stackrel{0}{\rightleftharpoons}$ & $\stackrel{\bullet}{=}$ & $\stackrel{0}{=}$ & $\stackrel{0}{\longrightarrow}$ & $\stackrel{6}{?}$ \\
\hline 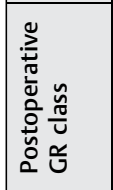 & $=$ & $=$ & $>$ & $>$ & $>$ & - & $=$ & $=$ & - & $>$ & $=$ & - \\
\hline 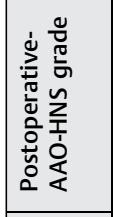 & $\infty$ & $\infty$ & 0 & 0 & 0 & $<$ & $\infty$ & $\infty$ & $\ll$ & 0 & $\infty$ & $\ll$ \\
\hline 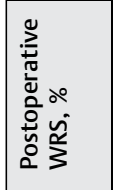 & $\infty$ & $\tilde{\sigma}$ & $\circ$ & 0 & 0 & $\stackrel{2}{\wedge}$ & ஃ & $\infty$ & 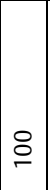 & 0 & $\infty$ & \% \\
\hline 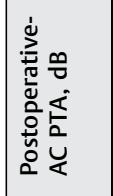 & $\stackrel{\infty}{m}$ & $\stackrel{\infty}{\sim}$ & $\infty$ & $\stackrel{\infty}{\infty}$ & $\stackrel{\stackrel{\sim}{\simeq}}{\simeq}$ & $\stackrel{\infty}{\sim}$ & $\approx$ & g & $\stackrel{\llcorner}{\sim}$ & $\stackrel{\Xi}{\rightleftarrows}$ & $\stackrel{\circ}{\circ}$ & $\infty$ \\
\hline 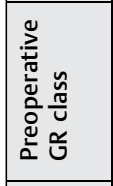 & $=$ & $=$ & $=$ & - & $\equiv$ & - & $=$ & $=$ & - & - & $=$ & - \\
\hline 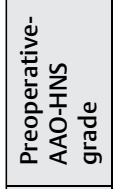 & $\infty$ & $\infty$ & $\infty$ & $<$ & $\cup$ & $<$ & $\infty$ & $\infty$ & $<$ & $\ll$ & $\infty$ & $<$ \\
\hline 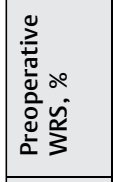 & $\tilde{\sigma}$ & ஃ & $\infty$ & \& & in & $\infty$ & 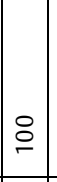 & 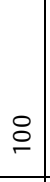 & 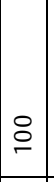 & 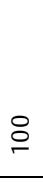 & $\stackrel{2}{2}$ & 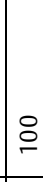 \\
\hline 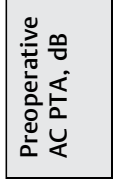 & $\tilde{m}$ & F & q & $\lesssim$ & t & $\stackrel{\sim}{\sim}$ & $\dot{m}$ & q & $\stackrel{ \pm}{\sim}$ & 0 & f & in \\
\hline 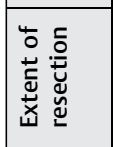 & 总 & 总 & 簿 & 兰 & 总 & 弟 & 总 & 弟 & 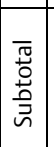 & $\stackrel{\varrho}{E}$ & 总 & 兰 \\
\hline 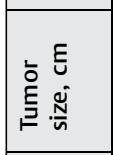 & \begin{tabular}{|l}
$g$ \\
$\dot{d}$ \\
$x$ \\
$m$ \\
$m$ \\
\end{tabular} & $\begin{array}{l}\stackrel{0}{\dot{-}} \\
\times \\
\stackrel{-}{-} \\
\end{array}$ & $\begin{array}{l}\stackrel{0}{\dot{x}} \\
\times \\
\stackrel{\circ}{i}\end{array}$ & $\begin{array}{l}n \\
0 \\
x \\
x \\
\stackrel{0}{-} \\
-\end{array}$ & $\begin{array}{l}\infty \\
0 \\
\dot{0} \\
0 \\
0 \\
- \\
\end{array}$ & $\begin{array}{l}\stackrel{0}{\dot{x}} \\
\dot{\sim} \\
\stackrel{-}{-}\end{array}$ & \begin{tabular}{|l|}
$\stackrel{0}{\dot{x}}$ \\
$\times$ \\
$\stackrel{\leftrightarrow}{r}$ \\
\end{tabular} & $\begin{array}{l}\stackrel{+}{+} \\
\dot{x} \\
\times \\
\infty \\
\stackrel{-}{-} \\
\end{array}$ & \begin{tabular}{|l|}
$\infty$ \\
0 \\
0 \\
$\times$ \\
0 \\
- \\
\end{tabular} & 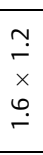 & 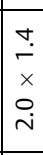 & $\begin{array}{l}\stackrel{\sim}{\tilde{x}} \\
\dot{\leftrightarrow} \\
\stackrel{\leftrightarrow}{\leftarrow} \\
\end{array}$ \\
\hline 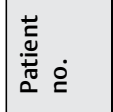 & - & $N$ & $m$ & $\nabla$ & in & 6 & $r$ & $\infty$ & $a$ & $\stackrel{\circ}{\circ}$ & $=$ & $\simeq$ \\
\hline
\end{tabular}


Table 5 Operative details

\begin{tabular}{|l|l|}
\hline Operative time, $\min$ & \\
\hline Mean & 261.6 \\
\hline Range & $180-512$ \\
\hline Estimated blood loss, $\mathrm{mL}$ & \\
\hline Mean & 56.25 \\
\hline Range & $50-100$ \\
\hline Length of hospital stay, d & \\
\hline 2 & 2 \\
\hline 3 & 5 \\
\hline 4 & 3 \\
\hline 5 & 1 \\
\hline 6 & 0 \\
\hline 7 & 1 \\
\hline Mean & 3.6 \\
\hline
\end{tabular}

or at follow-up. One patient was found to have a recurrent tumor laterally in the IAC. This patient was offered radiosurgery but declined. He was subsequently offered hearing preservation surgery via the middle fossa approach but again declined. Ultimately this patient chose to undergo a translabyrinthine approach for gross total resection of the recurrent tumor.

\section{Discussion}

Although the surgical endoscope was first introduced to CPA surgery in $1917,{ }^{2}$ the operative microscope has remained the standard approach. Historically, this was due to the poor magnification and illumination that the endoscope provided. When the operative microscope was introduced in the 1960s, it proved superior to the endoscope in these ways. Microscopy alone, however, was not without its limitations in posterolateral skull base surgery. The linear light source, located outside the surgical field, created many dark recesses due to the cavernous nature of the CPA and the various structures that reside in it. Although some of these corners could be visualized with larger cranial openings, various degrees of cerebellar retraction, or manipulation of neurovascular structures, these maneuvers can increase morbidity while still leaving many areas suboptimally illuminated, potentially leading to inadvertent injury to adjacent structures.

Technological improvements in image resolution and lighting allowed for the reintroduction and renewed popularity of the surgical endoscope in skull base surgery throughout the 1990s. Logistical concerns, however, have significantly limited the use of the endoscope in the posterolateral skull base. The delicate, yet vital, structures of the skull base demand exact precision to avoid catastrophic complications. As a result, surgeons generally require bimanual dexterity to navigate these intricate corridors. In the endonasal and intraventricular approaches, this is easily attained by utilizing an assistant surgeon because the operative field can comfortably fit two surgeons and four hands working in unison. The small working area of the retrosigmoid lateral suboccipital area creates a difficult scenario for harmonious interaction between two surgeons. As a result of this impediment, the endoscope was initially relegated to an adjunct, used only to inspect the area of interest before and after the procedure was performed. This technique, however, was used by several authors to describe the superiority of endoscopic visualization in comparison with microscopy in the CPA, ${ }^{3-9}$ demonstrating the potential benefits of the endoscope and furthering motivation to overcome the existing logistical barriers.

Utilizing the findings of previous authors, several surgeons began implementing the endoscope into CPA surgery via the endoscopic-assisted technique. Reports of this procedure demonstrated successful results in microvascular decompression, ${ }^{17-25}$ vestibular neurectomy, ${ }^{23,26,27}$ and resection of various tumors such as epidermoid cysts ${ }^{28}$ and vestibular schwannomas. ${ }^{23,29-31}$ The endoscope allowed for full visualization of the CPA from cranial nerve IV at the level of the incisura to the foramen magnum as well as from the cerebellar hemisphere to the petrous portion of the temporal bone. In addition, angled endoscopes, such as the 30-degree scope, allowed for improved visualization into the IAC. Despite these improvements, the primary portions of these surgeries continued to be performed under microscopy because no appropriate endoscopic holding existed. This meant that suboptimal visualization was still being utilized during the most critical and dangerous portions of these procedures. In addition, significant cerebellar retraction, more soft tissue dissection and a larger craniotomy were necessary, all of which increase potential morbidity.

The introduction of polyaxial pneumatic holding arms have allowed for the next step in endoscopic posterolateral skull base surgery to occur. These arms have the ability to hold the endoscope rigidly in place, allowing the surgeon to operate with bimanual dexterity while still allowing optimal working space ( - Fig. 7). In addition, the holding arm is able to be easily moved in all planes of motion with the depression of a single button and reassume a rigid position with the release of the same button. As a result, fully endoscopic techniques were developed, allowing surgeons to optimize visualization, lessen cranial openings, eliminate cerebellar retraction, and minimize manipulation of neurovascular structures. Jarrahy et $\mathrm{al}^{13}$ were the first to describe a fully endoscopic approach for microvascular decompression. Since then, several authors have reported excellent results, both in clinical outcomes and recovery times. ${ }^{10-15}$ Reports of tumor resection, although scarce, are present in the literature. ${ }^{32}$ De Divitiis et al $^{33}$ and later Krass et $\mathrm{al}^{34}$ reported success in the removal of epidermoid tumors utilizing this technique. Shahanian et $\mathrm{al}^{35,36}$ have published successful results in the fully endoscopic approach to vestibular schwannomas; however, no other authors, to our knowledge, have reported data on this technique.

In our experience, the fully endoscopic technique can be used successfully for hearing preservation surgery for vestibular schwannomas. Not only was gross total resection possible for all patients, but 92\% also maintained a $\mathrm{HB}$ score of 


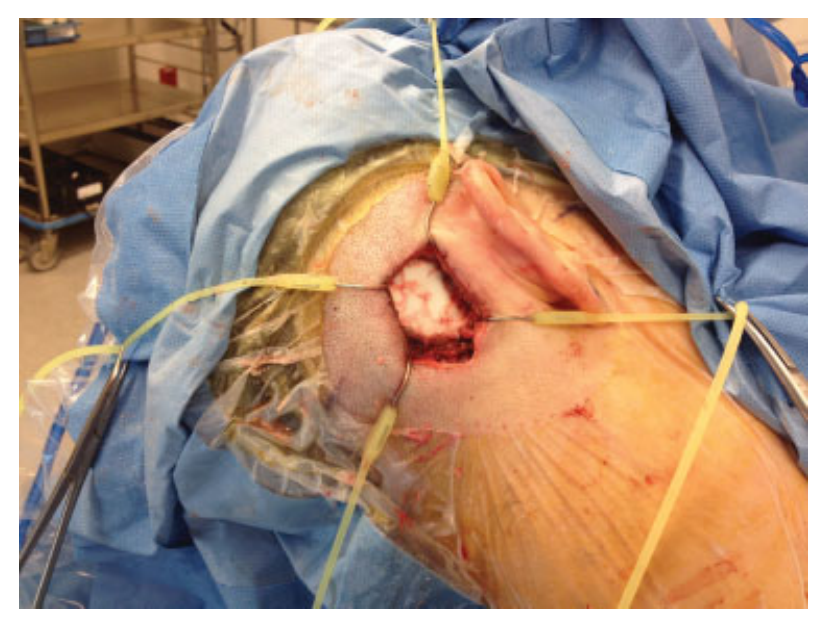

Fig. 7 Operative setup with the holding arm arching over the patient and rigidly holding the endoscope in place. Note how the surgeon is able to operate with bimanual dexterity while still maintaining optimal visualization.

1/6 postoperatively, demonstrating excellent facial nerve preservation. In addition, a hearing preservation rate of $67 \%$ is similar or better than most literature to date.

Potentially more importantly, our complication rate was $0 \%$ with no patients experiencing postoperative CSF leak, wound infection, meningitis, or cranial nerve palsy. Most of the patients were discharged home by postoperative day 4 , with a mean length of hospital stay of 3.6 days, again on par or better than most reports. Operative blood loss was also extremely low with a mean of only $56.3 \mathrm{~mL}$. Although our data did demonstrate overall successful results of the purely endoscopic technique, it must be acknowledged that our patient total of 12 , is relatively small, and all had small or medium size vestibular schwannomas that were primarily situated in the CPA.

Despite its advantages, limitations do exist in the purely endoscopic approach. Preoperative imaging must be carefully reviewed to ensure that the prepontine or interpeduncular cisterns are present. Drainage of these cisterns during surgery is key to obtain appropriate cerebellar relaxation, without which visualization is significantly limited. Heat from the endoscopic light source has the potential to cause thermal damage to surrounding structures, creating a significant hazard. To avoid this, we utilize a thin sleeve over the endoscope and intermittent irrigation to control high temperatures. Instrumentation is another struggle to overcome. The small working corridor that exists between the cerebellum and petrous temporal bone makes using the traditional bayonet-style instruments difficult, if not impossible. Instead, we use a separate instrument set that is based on a rotational pistol-grip style ( - Fig. 8). More recently, significant improvements in tumor aspirators and drilling systems designed specifically for endoscopic approaches have further improved tumor resections in the CPA. Most of these challenges can be easily overcome with proper preparation, but the main obstacle most skull base surgeons face is the steep learning curve required for posterolateral endoscopy. The endoscope forces surgeons to work in parallel to the endoscope, rather than the traditional manner of working in series along the

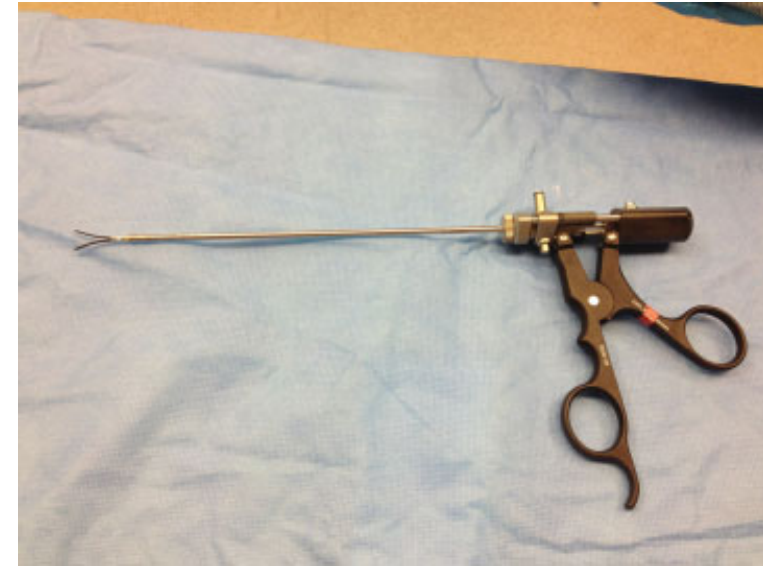

Fig. 8 Pistol-gripped bipolar that has replaced the bayonet-style traditional bipolar.

trajectory of the microscope. To alleviate this obstacle, we recommend that skull base surgeons have significant familiarity with the endoscope before attempting approaches to the CPA. Experience in cadaveric specimens or via other approaches, such as endonasal or intraventricular, is vital to flatten this learning curve.

\section{Conclusion}

Endoscopy alone, without the need for the operative microscope, can be safely used in hearing preservation surgery for vestibular schwannomas. Facial nerve and hearing preservation is similar if not improved compared with other approaches while providing a smaller incision, lesser cranial opening, no cerebellar retraction, and less manipulation of the neurovascular structures. The improved visualization of the endoscope is ideal for operating in the intricate corridors of the CPA, especially when dealing with complex pathology such as vestibular schwannomas. It is imperative, however, that surgeons are very comfortable with the endoscope and CPA anatomy before attempting such a procedure.

\section{Acknowledgments}

We thank Beverly Walters, MD, for her ongoing support and for editing this manuscript.

\section{References}

1 Mouton WG, Bessell JR, Maddern GJ. Looking back to the advent of modern endoscopy: 150th birthday of Maximilian Nitze. World J Surg 1998;22(12):1256-1258

2 Doyen E. Surgical Therapeutics and Operative Techniques. Vol 1. London, England: Balliere, Tindall, and Cox; 1917:599-602

3 Borucki L, Szyfter W, Leszczyńska M. Microscopy and endoscopy of the cerebellopontine angle in the retrosigmoid approach [in Polish]. Otolaryngol Pol 2004;58(3):509-515

4 Cappabianca P, Cavallo LM, Esposito F, de Divitiis E, Tschabitscher M. Endoscopic examination of the cerebellar pontine angle. Clin Neurol Neurosurg 2002;104(4):387-391 
5 Magnan J, Chays A, Lepetre C, Pencroffi E, Locatelli P. Surgical perspectives of endoscopy of the cerebellopontine angle. Am J Otol 1994;15(3):366-370

6 O'Donoghue GM, O'Flynn P. Endoscopic anatomy of the cerebellopontine angle. Am J Otol 1993;14(2):122-125

7 Takemura Y, Inoue T, Morishita T, Rhoton AL Jr. Comparison of microscopic and endoscopic approaches to the cerebellopontine angle. World Neurosurg 2014;82(3-4):427-441

8 Van Rompaey J, Bush C, McKinnon B, Solares AC. Minimally invasive access to the posterior cranial fossa: an anatomical study comparing a retrosigmoidal endoscopic approach to a microscopic approach. J Neurol Surg A Cent Eur Neurosurg 2013;74(1):1-6

9 Wackym PA, King WA, Meyer GA, Poe DS. Endoscopy in neurootologic surgery. Otolaryngol Clin North Am 2002;35(2):297-323

10 Artz GJ, Hux FJ, Larouere MJ, Bojrab DI, Babu S, Pieper DR. Endoscopic vascular decompression. Otol Neurotol 2008;29(7):995-1000

11 Eby JB, Cha ST, Shahinian HK. Fully endoscopic vascular decompression of the facial nerve for hemifacial spasm. Skull Base 2001; 11(3):189-197

12 Halpern $\mathrm{CH}$, Lang SS, Lee JY. Fully endoscopic microvascular decompression: our early experience. Minim Invasive Surg 2013;2013:739432

13 Jarrahy R, Eby JB, Cha ST, Shahinian HK. Fully endoscopic vascular decompression of the trigeminal nerve. Minim Invasive Neurosurg 2002;45(1):32-35

14 Setty P, Volkov AA, D’Andrea KP, Pieper DR. Endoscopic vascular decompression for the treatment of trigeminal neuralgia: clinical outcomes and technical note. World Neurosurg 2014;81(3-4): 603-608

15 Yadav YR, Parihar V, Agarwal M, Sherekar S, Bhatele P. Endoscopic vascular decompression of the trigeminal nerve. Minim Invasive Neurosurg 2011;54(3):110-114

16 American Academy of Otolaryngology-Head and Neck Surgery. Committee on Hearing and Equilibrium guidelines for the evaluation of hearing preservation in acoustic neuroma (vestibular schwannoma). American Academy of Otolaryngology-Head and Neck Surgery Foundation, Inc. Otolaryngol Head Neck Surg 1995; 113(3):179-180

17 Broggi M, Ferroli P, Acerbi F, Tringali G, Franzini A, Broggi G. The value of endoscopy in microvascular decompression procedures. Neurosurgery 2012;71(2):E564

18 Chen MJ, Zhang WJ, Yang C, Wu YQ Zhang ZY, Wang Y. Endoscopic neurovascular perspective in microvascular decompression of trigeminal neuralgia. J Craniomaxillofac Surg 2008;36(8):456-461

19 Duntze J, Litré CF, Eap C, et al. Adjunctive use of endoscopy during microvascular decompression in the cerebellopontine angle: 27 case reports [in French]. Neurochirurgie 2011;57(2):68-72

20 El-Garem HF, Badr-El-Dine M, Talaat AM, Magnan J. Endoscopy as a tool in minimally invasive trigeminal neuralgia surgery. Otol Neurotol 2002;23(2):132-135
21 Jarrahy R, Berci G, Shahinian HK. Endoscope-assisted microvascular decompression of the trigeminal nerve. Otolaryngol Head Neck Surg 2000;123(3):218-223

22 King WA, Wackym PA, Sen C, Meyer GA, Shiau J, Deutsch H. Adjunctive use of endoscopy during posterior fossa surgery to treat cranial neuropathies. Neurosurgery 2001;49(1):108-115; discussion $115-116$

23 Miyazaki H, Deveze A, Magnan J. Neuro-otologic surgery through minimally invasive retrosigmoid approach: endoscope assisted microvascular decompression, vestibular neurotomy, and tumor removal. Laryngoscope 2005;115(9):1612-1617

24 Rak R, Sekhar LN, Stimac D, Hechl P. Endoscope-assisted microsurgery for microvascular compression syndromes. Neurosurgery 2004;54(4):876-881; discussion 881-883

25 Teo C, Nakaji P, Mobbs RJ. Endoscope-assisted microvascular decompression for trigeminal neuralgia: technical case report. Neurosurgery 2006;59(4, Suppl 2):E489-E490; discussion E490

26 Cutler AR, Kaloostian SW, Ishiyama A, Frazee JG. Two-handed endoscopic-directed vestibular nerve sectioning: case series and review of the literature. J Neurosurg 2012;117(3):507-513

27 Wackym PA, King WA, Barker FG, Poe DS. Endoscope-assisted vestibular neurectomy. Laryngoscope 1998;108(12):1787-1793

28 Schroeder HWS, Oertel J, Gaab MR. Endoscope-assisted microsurgical resection of epidermoid tumors of the cerebellopontine angle. J Neurosurg 2004;101(2):227-232

29 Goksu N, Bayazit Y, Kemaloglu Y. Endoscopy of the posterior fossa and endoscopic dissection of acoustic neuroma. Neurosurg Focus 1999;6(4):e15

30 Magnan J, Chays A, Cohen JM, Caces F, Locatelli P. Endoscopy of the cerebellopontine angle. Rev Laryngol Otol Rhinol (Bord) 1995; 116(2):115-118

31 Wackym PA, King WA, Poe DS, et al. Adjunctive use of endoscopy during acoustic neuroma surgery. Laryngoscope 1999;109(8): 1193-1201

32 Pieper DR. The endoscopic approach to vestibular schwannomas and posterolateral skull base pathology. Otolaryngol Clin North Am 2012;45(2):439-454, $x$

33 de Divitiis O, Cavallo LM, Dal Fabbro M, Elefante A, Cappabianca P. Freehand dynamic endoscopic resection of an epidermoid tumor of the cerebellopontine angle: technical case report. Neurosurgery 2007;61(5, Suppl 2):E239-E240; discussion E240

34 Krass J, Hahn Y, Karami K, Babu S, Pieper DR. Endoscopic assisted resection of prepontine epidermoid cysts. J Neurol Surg A Cent E Neurosurg 2014;75(2):120-125

35 Shahinian HK, Eby JB, Ocon M. Fully endoscopic excision of vestibular schwannomas. Minim Invasive Neurosurg 2004;47(6): 329-332

36 Shahinian HK, Ra Y. 527 fully endoscopic resections of vestibular schwannomas. Minim Invasive Neurosurg 2011;54(2):61-67 